\title{
Magic Lenses for Revealing Device Interactions in Smart Environments (demo abstract)
}

\author{
Simon Mayer \\ ETH Zurich \\ simon.mayer@inf.ethz.ch \\ Yassin Nasir Hassan \\ ETH Zurich \\ hassanyestudent.ethz.ch
}

\author{
Gábor Sörös \\ ETH Zurich \\ gabor.soroseinf.ethz.ch
}

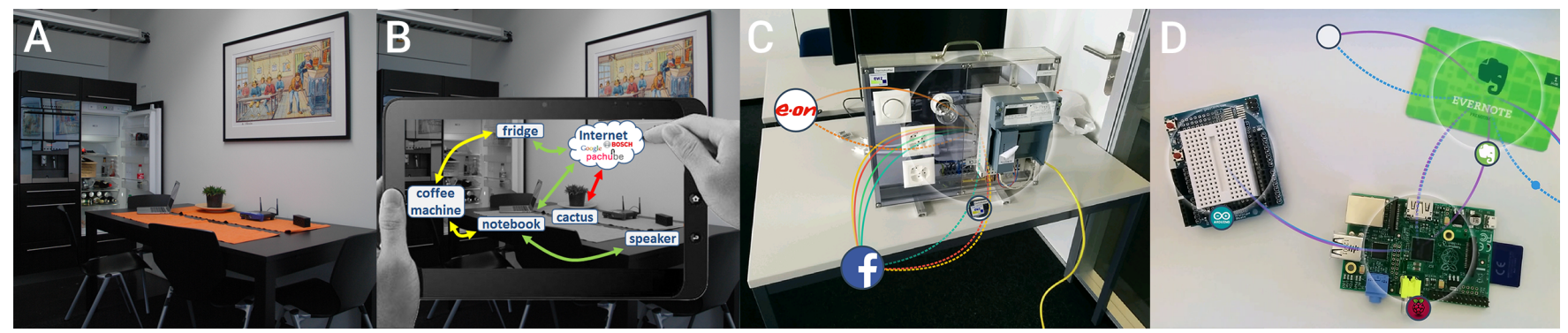

Figure 1: A magic lens for visualizing communication links in smart environments. A) and B) Idea: Users can reveal communication links by observing devices through a tablet. C) and D) Screenshots of our tool showing messages being passed between connected objects. The smart devices are recognized based on their visual features and their communication traces are overlaid on the screen.

\begin{abstract}
We present a tool for visualizing device interactions in smart environments as a magic lens by augmenting the live camera view of a tablet with relevant connections between recognized devices in the camera's field of view.
\end{abstract}

Keywords: Smart Environment, Web of Things, Network Management, Visualization, Augmented Reality

\section{Introduction}

As an ever increasing number of smart objects get connected to the Web of Things, users can easily lose track of interactions between the devices in their environment. We expect that devices in future smart homes such as refrigerators, stereo sets, and other domestic appliances will not only interact with each other and with user interface devices, but also with services that are hosted remotely by utility companies, manufacturers, or firms that analyze a household data to provide advanced services to inhabitants. This development also gives rise to a number of challenges that, if not properly addressed, might hinder the widespread adoption of smart homes because people could lose trust in the smart things and the remote services these things use [Brush et al. 2011].

\section{Contribution}

Our contribution is a tool for collecting and visualizing device interactions in smart home and office scenarios. The tool consists of a real-time logger that records application layer (HTTP) interactions between devices, a storage back-end to persist recorded messages, and a magic lens-like [Bier et al. 1993] handheld user interface. We propose to use object recognition technologies and methods known from the domain of augmented reality to take network monitoring to the next level, by visualizing data about connections between devices and services on mobile devices as an overlay for the physical world.

Our handheld augmented reality application recognizes smart devices based on their visual features and visualizes their interactions on top of the camera view of a tablet computer (see Fig. 1). This approach could allow users to easily and intuitively monitor the information flows between nodes inside their home networks and with remote services and thereby get more in control of their network installations - a similar idea has already been applied for visualizing urban infrastructures, for instance in the Vidente project [Schall et al. 2013] where underground tubing is visualized using an augmented reality application on a smartphone.

Our approach should enable users to better understand device associations in their smart home and to perceive unwanted interactions, where we aim to go beyond currently available network monitoring approaches whereas our method also allows to inspect the content of the HTTP traffic between devices.

\section{Demo Applications}

We envision the proposed system to be applicable in a number of use cases that range from educating students about distributed algorithms to enabling inhabitants of smart homes to track what kind of information is leaving the domestic environment, and where control commands to their devices come from. In particular, we have created demos with a smart electricity meter (Fig. 1C), with various connected development boards (Fig. 1D) and cars connected to the CloudThink (http://cloud-think.com) platform.

\section{References}

Bier, E. A., Stone, M. C., Pier, K., BuXton, W., And DeRose, T. D. 1993. Toolglass and Magic Lenses: The Seethrough Interface. In Proc. SIGGRAPH, ACM.

Brush, A. J., Lee, B., Mahajan, R., Agarwal, S., Saroiu, S., AND DIXON, C. 2011. Home Automation in the Wild: Challenges and Opportunities. In Proc. CHI, ACM.

Schall, G., Zollmann, S., And Reitmayr, G. 2013. Smart vidente: Advances in mobile augmented reality for interactive visualization of underground infrastructure. Personal Ubiquitous Comput. 17, 7 (Oct.), 1533-1549. 